\title{
SUBGROUPS OF FINITELY PRESENTED METABELIAN GROUPS
}

\author{
Dedicated to the memory of Hanna Neumann
}

GILBERT BAUMSLAG

(Received 9 May 1972)

Communicated by M. F. Newman

\section{Introduction}

In 1961 Graham Higman [1] proved that a finitely generated group is a subgroup of a finitely presented group if, and only if, it is recursively presented. Therefore a finitely generated metabelian group can be embedded in a finitely presented group.

The object of this paper is to prove the following:

THEOREM. A finitely generated metabelian group can be embedded in a finitely presented metabelian group.

This theorem may be viewed as an analogue of Higman's theorem for the variety of all metabelian groups. It suggests that the theory of finitely presented solvable groups may be far richer than one might have thought. It also seems likely that there are other varietal analogues of Higman's theorem.

There is another facet of this theorem that is worth commenting on. For some time now the complexity of the abelian normal subgroup structure of finitely presented groups has been up in the air. Indeed a finitely presented group with a free abelian normal subgroup of infinite rank has only very recently been constructed (Baumslag [3]). The existence of such complex finitely presented groups is reinforced by our theorem. For it follows from it that the abelian normal subgroup structure of finitely presented metabelian groups is, in a sense, as complicated as the abelian normal subgroup structure of finitely generated metabelian groups.

\section{Sketch of the proof of the theorem}

Let $G$ be a finitely generated metabelian group. Our objective is to embed $G$ in a finitely presented metabelian group.

Support from the National Science Foundation Grant is gratefully acknowledged. 
The first step in the proof is to embed $G$ in a factor group $W / N$ of the wreath product $W$ of two finitely generated abelian groups. This embedding can be accomplished in many ways. Here we shall use a theorem of Magnus [4] which makes it easy to arrange that $N$ be contained in the base group of $W$, a crucial factor in the proof.

The second step is to provide a procedure for embedding $W$ in a finitely presented metabelian group $W^{*}$. This procedure, which is the main part of the proof, has a certain degree of flexibility which we exploit in the last part of the proof.

The third and final step in the proof is to show that $W / N$ (and hence $G$ ) can be embedded in a suitable quotient group $E$ of $W^{*}$. Now finitely generated metabelian groups satisfy the maximal condition for normal subgroups (Hall [2]). It follows that every factor group of a finitely presented metabelian group is finitely presented, and so in particular $E$ is finitely presented, as desired.

\section{Preliminaries}

In this section we record some of the notation and notions we shall need in the sequel.

As usual if $x, y$ are elements of a group $G$ and $M$ is a subgroup of $G$ then we define

$$
x^{y}=y^{-1} x y,[x, y]=x^{-1} y^{-1} x y \text { and } M^{x}=x^{-1} M x .
$$

We denote the integral group ring of the group $G$ by $\mathbb{Z} G$ and write $n$ in place of $n \cdot 1$, where 1 is the unit element of $G$, for each integer $n$. If $a \in G$ and $f \equiv n_{1} g_{1}$ $+\cdots+n_{k} g_{k}$ is a specific expression for an element in the group ring $\mathbb{Z} G$ then we define

$$
a^{f}=\left(a^{n_{1}}\right)^{g_{1}}\left(a^{n_{2}}\right)^{g_{2}} \cdots\left(a^{n_{k}}\right)^{g_{k}}
$$

(where here $n_{1}, \cdots, n_{k}$ are integers and $g_{1}, \cdots, g_{k} \in G$ ). If $M$ is an abelian normal subgroup of $G$ and $T$ is some other subgroup of $G$, then $M$ becomes a $\mathbb{Z} T$-module by defining

$$
a f=a^{f}(a \in M, f \in \mathbb{Z} T) .
$$

We recall that a group $W$ is the (standard) wreath product of its subgroups $A$ and $T$ if

(i) $W=g p(A, T)$

(ii) $B=g p_{W}(A)$, the normal closure of $A$ in $W$, is the direct sum of its subgroups $A^{t}(t \in T)$.

Notice that (ii) implies that $B \cap T=1 . B$ is called the base group of $W$, which is usually denoted by $A<T$.

The proof of the following lemma is straightforward and is therefore omitted. 
LEMMA. 1. Let $w, x, y$ be elements of a group.

(i) If $[x, y]=1$ then $\left[x^{w}, y^{w}\right]=1$.

(ii) If $[x, w]=[x, y]=1$, then $[w x, y]=[w, y]=[w, x y]$.

A finite subset $\left\{t_{1}, \cdots, t_{n}\right\}$ of non-identity elements of a finitely generated abelian group $T$ is termed a basis of $T$ if $T$ is the direct sum of its subgroups $g p\left(t_{i}\right)(i=1, \cdots, n)$.

\section{Some simple notions from commutative ring theory}

We record here some simple constructive techniques which will play an important role in the proof of our theorem (see e.g. Atiyah and Macdonald [5], Chapter. 3). To this end, let $R$ be a commutative ring with 1 and let $S$ be a multiplicatively closed subset of $R$ (containing 1 ). Let $\sim$ be the equivalence relation in $R \times S$ defined as follows:

$$
(r, s) \sim\left(r^{\prime} s^{\prime}\right) \text { if there exists } t \in S \text { such that }\left(r s^{\prime}-s r^{\prime}\right) t=0 .
$$

We denote the set of equivalence classes in $R \times S$ by $R S^{-1}$ and the equivalence class containing $(r, s)$ by $r s^{-1} \cdot R S^{-1}$ is turned into a ring by defining addition and multiplication in the usual way:

$$
r s^{-1} \cdot r_{1} s^{-1}=r r_{1}\left(s s_{1}\right)^{-1}, r s^{-1}+r_{1} s_{1}^{-1}=\left(r s_{1}+s r_{1}\right)\left(s s_{1}\right)^{-1} \text {. }
$$

Suppose that there are no zero divisors (of $R$ ) in $S$; i.e., suppose

$$
a b=0 \text { implies } a=0 \text { whenever } a \in R, b \in S \text {. }
$$

Then the mapping

$$
r \mapsto r 1^{-1}
$$

is an injection (i.e. a monomorphism) of $R$ into the ring $R S^{-1}$. Abusing the notation we have just introduced we denote $r 1^{-1}$ by $r$ and $1 s^{-1}$ by $s^{-1}$.

There is a similar construction for modules. In order to explain, let $M$ be an $R$-module. We define an equivalence relation $\sim$ on $M \times S$ as follows:

$$
(m, s) \sim\left(m^{\prime}, s^{\prime}\right) \text { only if there exists } t \in S \text { such that }\left(m s^{\prime}-m^{\prime} s\right) t=0 .
$$

We denote the equivalence class of $(m, s)$ by $m s^{-1}$ and the set of such equivalence classes by $M S^{-1} . M S^{-1}$ may be turned into an $R S^{-1}$-module by first defining

$$
m s^{-1}+m_{1} s_{1}^{-1}=\left(m s_{1}+m_{1} s\right)\left(s s_{1}\right)^{-1}
$$

and then defining the action of $R S^{-1}$ on $M S^{-1}$ by

$$
\left(m s^{-1}\right)\left(r t^{-1}\right)=m r(s t)^{-1}(m \in M, r \in R, s, t \in S) \text {. }
$$

If the condition (1) together with the following condition 


$$
m s=0 \text { implies } m=0(m \in M, s \in S)
$$

are both satisfied, then the mapping

$$
m \mapsto m 1^{-1}
$$

is a monomorphism of the $R$-module $M$ into the $R$-module $M S^{-1}$ (where $M S^{-1}$ is an $R$-module in the obvious way). Finally we remark that, when convenient, we denote $m 1^{-1}$ simply by $m$.

\section{The first step in the proof of the theorem}

We shall need the following theorem of Magnus [4], which we record here as Lemma 2.

LEMMA 2. Let $F$ be a free group, freely generated by a set $X=\left\{x_{i} \mid i \in I\right\}$, and let $R$ be a normal subgroup of $F$. Let the mapping $x_{i} R \mapsto t_{i}(i \in I)$ define an isomorphism from $F / R$ to a group $T=g p\left(t_{i} \mid i \in I\right)$. Furthermore, let $A$ be a free abelian group, freely generated by the elements $a_{i}(i \in I)$. Then the mapping

$$
x_{i}[R, R] \mapsto a_{i} t_{i}(i \in I)
$$

defines an isomorphism of $F /[R, R]$ into $W=A<T$ (where $[R, R]$ is the derived group of $R$ ).

We use Lemma 2 to prove

LEMMA 3. Let $G$ be a finitely generated metabelian group. Then there exists a free abelian group $A$ of finite rank and a finitely generated abelian group $T$ such that $G$ is isomorphic to a subgroup of $W / N$, where $W=A \geq T$ and $N$ is a normal subgroup of $W$ contained in the base group.

Proof. Let $\left\{g_{1}, g_{2}, \cdots, g_{n}\right\}$ be a finite set of generators of $G$, let $F$ be the free group, freely generated by $x_{1}, x_{2}, \cdots x_{n}$ and let $\phi$ be the epimorphism from $F$ to $G$ defined by

$$
\phi: x_{i} \mapsto g_{i}(i=1,2, \cdots, n) .
$$

Let $K$ be the kernel of $\phi$ and let $R$ be the inverse image of $[G, G]$ under $\phi$. Then $R / K \cong[G, G]$ and hence is abelian. Therefore $[R, R] \leqq K$, which means that

$$
[R, R] \leqq K \leqq R
$$

Now let $A$ be a free abelian group on $a_{1}, a_{2}, \cdots, a_{n}$ and let $W=A 2 T$, where $T=F / R$. Let $\psi$ be the homomorphism of $F$ into $W$ defined by

$$
x_{i} \psi=a_{i} t_{i}\left(t_{i}=x_{i} R, i=1, \cdots, n\right) .
$$

By Lemma 2 the kernel of $\psi$ is $[R, R]$. Hence $\psi$ induces an isomorphism $\psi_{*}$, say, of $F /[R, R]$ into $W$. 
Let us now put $N=(K /[R, R]) \psi_{*}$ (see (3)). Now $N \leqq(F /[R, R]) \psi_{*}$. Therefore $N$ is normalised by the elements $a_{i} t_{i}, i=1,2, \cdots, n$. But it follows from the definition of $\psi_{*}$ that $N$ is contained in the base group $B$ of $W$. Since $B$ is abelian $N$ is normalised (indeed centralised) by $B$ and hence by the elements $t_{i}$, and therefore by all of $W$. In other words $N$ is normal in $W$. Therefore $G$ has been embedded in $W / N-$ it is isomorphic to $(F /[R, R]) \psi_{*} / N$. This completes the proof of Lemma 3 and with it the first step in the proof of the theorem.

\section{Embedding wreath products in finitely presented groups}

This section prepares the way for embedding certain quotient groups of wreath products in finitely presented metabelian groups. This embedding procedure will be completed in the next section.

Let $A$ be a free abelian group of finite rank freely generated by the elements

$$
a_{1}, a_{2}, \cdots, a_{r}
$$

Furthermore let $T$ be a finitely generated abelian group with basis

$$
t_{1}, t_{2}, \cdots, t_{k}, \cdots, t_{l},
$$

where $t_{1}, t_{2}, \cdots, t_{k}$ are of infinite order and $t_{k+1}, \cdots, t_{l}$ are respectively of finite order $e_{k+1}, \cdots, e_{l}$. Finally let

$$
F=\left\{f_{1}, f_{2}, \cdots, f_{k}\right\}
$$

be a set of element $f_{i}$ of the group ring $\mathbb{Z} T$ of $T$, where each $f_{i}$ is of the form

$$
f_{i}=1+c_{i, 1} t_{i}+c_{i, 2} t_{i}{ }^{2}+\cdots+c_{i, d_{i}-1} t_{i}^{d_{i}-1}+t_{i}^{d_{i}}\left(d_{i} \geqq 1\right)\left(c_{i, j} \in \mathbb{Z}\right)
$$

We embed $W=A \nmid T$ in a finitely presented metabelian group $W_{F}$, which, as the notation suggests, depends not only on $W$ but also on $F$.

We shall present $W_{F}$ on the generators

$$
X=\left\{a_{1}, a_{2}, \cdots, a_{r}, t_{1}, t_{2}, \cdots, t_{l}, u_{1}, u_{2}, \cdots, u_{k}\right\},
$$

where the integers $r, k$ and $l$ are the integers occurring in (4) and (5).

The defining relations of $W_{F}$ are of four kinds. First we have the power relations

$$
t_{i}^{e_{i}}=1 \quad(i=k+1, \cdots, l)
$$

Next we have the commutativity relations

$$
\begin{cases}{\left[u_{i}, u_{j}\right]=1} & (1 \leqq i, j \leqq k) \\ {\left[t_{i}, t_{j}\right]=1} & (1 \leqq i, j \leqq l) \\ {\left[t_{i}, u_{j}\right]=1} & (1 \leqq i \leqq l, 1 \leqq j \leqq k) \\ {\left[a_{i}, a_{j}\right]=1} & (1 \leqq i, j \leqq r) .\end{cases}
$$


Thirdly we have the commutativity relations for the conjugates of the generator $a_{i}$ :

$$
\left\{\begin{array}{l}
{\left[a_{i}^{v}, a_{j}^{w}\right]=1 \text { where } 1 \leqq i, j \leqq r \text { and }} \\
v, w \in\left\{t_{1}^{\alpha(1)} t_{2}^{\alpha(2)} \cdots t_{l}^{\alpha(l)} \mid 0 \leqq \alpha(i) \leqq d_{i}\right. \\
\left.0 \leqq \alpha(i)<e_{i}(i=k+1, \cdots, l)\right\}
\end{array} \quad(i=1,2, \cdots, k)\right.
$$

Notice that the positive integer $d_{i}$ occurring in (11) is simply the "degree" of the "polynomial" $f_{i}$ (cf (7)).

Finally we have the defining relations giving the action of the elements $u_{j}$ on the elements $a_{i}$ :

$$
a_{i}^{u j}=a_{i}^{f_{j}} \quad(1 \leqq i \leqq r, 1 \leqq j \leqq k),
$$

where the $f_{j}$ are again the elements comprising $F$ (see (6), (7)).

We emphasise that $W_{F}$ is the group generated by the set $X$ given by (8) subject to the defining relations (9), (10), (11), and (12). $W_{F}$ is patently finitely presented. We shall prove that $W_{F}$ is also metabelian and in the next section, that $W$ is embedded in $W_{F}$. The main difficulty is the proof that $W_{F}$ is metabelian. The crucial step in this proof is contained in the following lemma, where we shall repeatedly make use of Lemma 1.

LeMma 4. Let $a, b, t, u$ be elements of a group $G$ and let $d$ be a positive integer. Suppose that

and that

$$
\left[a^{v}, b^{w}\right]=1 \text { whenever } v, w \in\left\{t^{i} \mid i=0,1, \cdots, d\right\}
$$

In addition suppose that

$$
[t, u]=1 \text {. }
$$

$$
a^{u}=a^{f}, b^{u}=b^{f}
$$

where

$$
f \equiv 1+c_{1} t+\cdots+c_{d-1} t^{d-1}+t^{d}
$$

is a specific expression for an element in the group ring $\mathbb{Z} G$. Then

$$
\left[a^{v}, b^{w}\right]=1 \text { whenever } v, w \in g p(t) \text {. }
$$

Proof. It follows easily from Lemma 1 that it suffices to prove that

$$
\left[a^{v}, b^{w}\right]=1 \text { whenever } v, w \in\left\{t^{i} \mid i \geqq 0\right\} \text {. }
$$

Suppose then that

$$
\left[a^{v}, b^{w}\right]=1 \text { whenever } v, w \in\left\{t^{i} \mid i=0,1, \cdots, m+d\right\} .
$$

We proceed by induction on $m$. When $m=0$ the relations (13) are part of the 
hypothesis. Let $n$ be a positive integer and assume that (13) holds whenever $m<n$. Then

$$
1=\left[a, b^{t^{n}}\right]=\left[a, b^{t^{n}}\right]^{u}=\left[a^{u}, b^{t^{n}}\right]=\left[a^{u}, b^{u t^{n}}\right]
$$

since $[t, u]=1$. But

$$
a^{u}=a^{f} \text { and } b^{u}=b^{f} \text {. }
$$

It follows that

$$
\left[a^{f}, b^{f t^{n}}\right]=1 \text {. }
$$

Observe that $a^{f}$ is a product of powers of elements of the form $a^{t^{i}}(i=0,1, \cdots, d)$ while $b^{f t^{n}}$ is itself a product of powers of elements of the form $b^{t^{t}}(i=n, n+1$, $\cdots, n+d)$. Indeed on noting that

$$
f=1+c_{1} t+\cdots+c_{d-1} t^{d-1}+t^{d}
$$

we find, on repeated application of Lemma 1 and the inductive assumption, that

$$
1=\left[a^{f}, b^{f t^{n}}\right]=\left[a, b^{t^{n+d}}\right] \text {. }
$$

If we consider now the commutator $\left[a^{t^{n}}, b\right]$ then it follows in precisely the same way that

$$
\left[a^{t^{n+d}}, b\right]=1
$$

Putting the inductive hypothesis together with (14) and (15) yields

$$
\left[a^{v}, b^{w}\right]=1\left(v, w \in\left\{t^{i} \mid i=0,1, \cdots, n+d\right\}\right) .
$$

This completes the inductive step and with it the proof of the lemma.

We make use now of Lemma 4 to prove

LEMMA 5. The finitely presented group $W_{F}$ is metabelian.

Proof. Let $M$ be the normal closure of $a_{1}, a_{2}, \cdots, a_{r}$ in $W_{F}$, i.e. the least normal subgroup of $W_{F}$ containing $a_{1}, a_{2}, \cdots a_{r}$. Now it follows from the definition (8) of $X$ and the defining relation (10) that $W_{F} / M$ is abelian. So it suffices to prove that $M$ is abelian.

It follows from (10) that $M$ is generated by the elements

$$
\left\{\begin{array}{c}
a_{i}^{v}\left(1 \leqq i \leqq r, v \in\left\{t_{1}^{\alpha(1)} t_{2}^{\alpha(2)} \cdots t_{l}^{\alpha(l)} u_{1}^{\beta(1)} u_{2}^{\beta(2)} \cdots u_{k}^{\beta(k)} \mid\right.\right. \\
\alpha(1), \cdots, \beta(k) \in \mathbb{Z}\})
\end{array}\right.
$$

So we have to prove that the elements in (16) commute. The elements $t_{i}$ commute with the elements $u_{j}$ (by (10)) and

$$
a_{i}^{u_{j}}=a_{i}^{f_{j}}
$$


where $f_{j}$ is a polynomial in $t_{j}$ (by (12)). It follows from these remarks and (i) of Lemma 1 that in order to prove that the elements in (16) commute it is enough to prove that the elements

$$
\begin{aligned}
& a_{i}^{v}\left(1 \leqq i \leqq r, v \in\left\{t_{1}^{\alpha(1)} t_{2}^{\alpha(2)} \cdots t_{l}^{\alpha(l)} \mid\right.\right. \alpha(1), \alpha(2), \cdots, \alpha(k) \in \mathbb{Z}, \\
&\left.\left.0 \leqq \alpha(i)<e_{i}(i=k+1, \cdots, l)\right\}\right)
\end{aligned}
$$

commute. Finally we observe that in order to prove that the elements in (17) commute, we need only prove, making use of the commutativity of the elements $t_{1}, t_{2}, \cdots, t_{l}$ and (i) of Lemma 1 , that the elements

$$
\left\{\begin{array}{c}
a_{i}^{v}\left(1 \leqq i \leqq r, v \in\left\{t_{1}^{\alpha(1)} \cdots t_{l}^{\alpha(l)} \mid \alpha(1), \alpha(2), \cdots, \alpha(k)\right.\right. \\
\left.\left.0 \leqq \alpha(i)<e_{i} \quad(i=k+1, \cdots, l)\right\}\right)
\end{array}\right.
$$

commute. In fact (18) follows easily by a repeated application of Lemma 4 . Indeed, in view of the defining relations (10) and (11) of $W_{F}$, Lemma 4 can be first applied to show the elements

$$
\begin{aligned}
a_{i}^{v}\left(1 \leqq i \leqq r, v \in\left\{t_{1}^{\alpha(1)} \cdots t_{k}^{\alpha(k)} \mid \alpha(1) \text { any integer, } 0 \leqq \alpha(i) \leqq d_{i}\right.\right. \\
\left.\left.(i=2, \cdots, k) 0<\alpha(i)<e_{i}(i=k+1, \cdots, l)\right\}\right)
\end{aligned}
$$

commute. We emphasise that here the first exponent $\alpha(1)$ is allowed to range over all the integers, but that the other exponents are still restricted. However on applying Lemma $4 k-1$ more times it follows that all of the elements in (18) commute, as required. This completes the proof of Lemma 5.

\section{Embedding wreath products in finitely presented groups}

Let $W=A \int T$ and $W_{F}$ be the groups defined in the preceding section. Furthermore let $B$ be the base group of $W$ and let $N$ be a normal subgroup of $W$ contained in $B . B / N$ is an abelian normal subgroup of $W / N$ and as such may be viewed as an $R(=\mathbb{Z} T)$-module. It is therefore convenient to put $M=B / N$ and to think of $M$ as an additive abelian group. Notice that $W / N$ is a split extension of $M$ by (a group isomorphic to) $T$ :

$$
W / N=M \cdot T, T \cap M=1 .
$$

Assume now that for each $f_{i} \in F$

$$
m f_{i}=0 \text { only if } m=0(m \in M) .
$$

We shall construct a group $E$ containing $W / N$ by using the ideas introduced in \$6. In particular this will enable us to prove that $W$ is embedded in $W_{F}$. To this end let $S$ be the multiplicatively closed set generated by $F \cup\{1\}$. Now $S$ contains 
no zero divisors of $R$. So $R$ is embedded in $R S^{-1}$ and the elements $f_{1}, f_{2}, \cdots, f_{k}$ are invertible in $R S^{-1}$ (see $\S 4$ ). It follows from (20) that if $m \in M$ and $s \in S$, then

$$
m s=0 \text { only if } m=0 .
$$

So if we think of the $R S^{-1}$ module $M S^{-1}$ simply as an $R$-module, the $R$-module $M$ is embedded in $M S^{-1}$.

Let us now put

$$
V=g p\left(T, f_{1}, \cdots, f_{k}\right)
$$

and form the split extension $E$ of $M S^{-1}$ by $V . E$ then consists of the pairs $(v, m)$ $\left(v \in V, m \in M S^{-1}\right)$ with multiplication defined by

$$
(v, m)\left(v^{\prime}, m^{\prime}\right)=\left(v v^{\prime}, m v^{\prime}+m^{\prime}\right)\left(v, v^{\prime} \in V, m, m^{\prime} \in M\right) .
$$

It follows from the fact that $W / N$ is a split extension of $M$ by $T$ (see (19)) and the fact that $M$, qua $R$-module, is embedded in $M S^{-1}$ qua $R$-module, that $W / N$ is embedded in $E$.

Let us now put

$$
a_{i}^{\prime}=\left(1, a_{i} N\right), t_{i}^{\prime}=\left(t_{i}, 0\right), u_{i}^{\prime}=\left(f_{i}, 0\right)
$$

(where we have used 0 in place of the zero element $N$ of $M(!)$ ). It follows from the very definition of $E$ that the defining relations for $W^{*}=W_{F}$, viz. (9), (10), (11), (12), hold also when $a_{i}, t_{i}, u_{i}$ are replaced by $a_{i}^{\prime}, t_{i}^{\prime}$, and $u_{i}^{\prime}$ respectively. Therefore the mapping of $X$ (see (8)) into $E$ defined by

$$
a_{i} \mapsto a_{i}^{\prime}, t_{i} \leftrightarrow t_{i}^{\prime}, u_{i} \leftrightarrow u_{i}^{\prime}
$$

determines a homomorphism, say $\psi$, of $W^{*}$ onto $E$. We are now in a position to prove

Lemma 6. $W$ is embedded in $W_{F}$.

Proof. Suppose that $N=1$. Then the condition (20) is certainly satisfied because $M$ is a free $R$-module in this case. Therefore the subgroup of $W_{F}$ generated by $a_{1}, a_{2}, \cdots, a_{r}, t_{1}, t_{2}, \cdots, t_{l}$ is mapped by $\psi$ onto an isomorphic copy of $W$ (because $W$ is embedded in $E$ ). On the other hand, there is a homomorphism $\psi^{\prime}$ of $W$ onto the above subgroup of $W_{F}$, since by Lemma 6 , the elements given in (17) commute, ensuring that the relations satisfied by $a_{1}^{\prime}, \cdots, a_{r}^{\prime}, t_{1}^{\prime}, \cdots, t_{l}^{\prime}$ are correspondingly satisfied by $a_{1}, \cdots, a_{r}, t_{1}, \cdots, t_{l}$ (thought of as elements of $W_{F}$ ). But it follows readily that $\psi^{\prime} \psi$ is the identity on $W$. Therefore $\psi^{\prime}$ induces an injection of $W$ into $W_{F}$, as desired.

This completes the second part of the proof. We shall return to the group $E$ and the normal subgroup $N$ of $W$ in $\S 9$ when the proof of the theorem itself is completed. 


\section{An explicit embedding of a free metabelian group in a finitely presented metablian group}

It is perhaps worth extracting an explicit embedding of the wreath product of two free abelian groups in a finitely presented metabelian group in order to obtain an explicit embedding of a free metabelian group in a finitely presented metabelian group. To this end let $T$ be a free abelian group of rank $r$ on $t_{1}, t_{2}, \cdots, t_{r}$ (i.e., here $k=r$ ) and let

$$
F=\left\{f_{1}=1+t_{1}, f_{2}=1+t_{2}, \cdots, f_{r}=1+t_{r}\right\} .
$$

Then $W_{F}$ is a $3 r$-generator group on $a_{1}, \cdots, a_{r}, t_{1}, \cdots, t_{r}, u_{1}, \cdots, u_{r}$ subject to the defining relations

$$
\left\{\begin{array}{c}
{\left[u_{i}, u_{j}\right]=\left[t_{i}, t_{j}\right]=\left[t_{i}, u_{j}\right]=\left[a_{i}^{v}, a_{j}^{w}\right]=1} \\
\left(1 \leqq i, j \leqq r, v, w \in\left\{t^{\alpha(1)} \cdots t^{\alpha(r)} \mid \alpha(1), \alpha(2), \cdots, \alpha(r) \in\{0,1\}\right\}\right)
\end{array}\right.
$$

and

$$
a_{i}^{u_{j}}=a_{i}^{1+t_{j}} \quad(1 \leqq i, j \leqq r) .
$$

By Magnus' theorem (Lemma 1) it follows that $g p\left(a_{1} t_{1}, a_{2} t_{2}, \cdots, a_{r} t_{r}\right)$ is a free metabelian group of rank $r$. So we have the following corollary of Lemma 6 viz.

COROLLARY 1. The finitely presented metabelian group with generators $a_{1}, \cdots, a_{r}, t_{1}, \cdots, t_{r}, u_{1}, \cdots, u_{r}$ subject to the finitely many defining relation (21) and (22) contains a free metabelian group of rank $r$.

In particular if $r=2$ we obtain the

Corollary 2. The free metabelian group of rank two can be embedded in a 6-generator 74-relator metabelian group $H$. The generators of $H$ may be chosen to be

$$
u_{1}, u_{2}, t_{1}, t_{2}, a_{1}, a_{2},
$$

while the 74 defining relations in terms of these generators may be chosen as

and

$$
\begin{gathered}
{\left[u_{1}, u_{2}\right]=\left[t_{1}, t_{2}\right]=\left[t_{1}, u_{1}\right]=\left[t_{1}, u_{2}\right]=\left[t_{2}, u_{1}\right]=\left[t_{2}, u_{2}\right]=1,} \\
{\left[a_{1}^{v}, a_{j}^{w}\right]=1\left(1 \leqq i, j \leqq 2, v, w \in\left\{t_{1}^{\alpha(1)} t_{2}^{\alpha(2)} \mid \alpha(1), \alpha(2) \in\{0,1\}\right\}\right)}
\end{gathered}
$$

$$
a_{1}^{u_{1}}=a_{1}^{1+t_{1}}, a_{1}^{u_{2}}=a_{1}^{1+t_{2}}, a_{2}^{u_{1}}=a_{2}^{1+t_{1}}, a_{2}^{u_{2}}=a_{2}^{1+t_{2}} .
$$

Actually Corollary 2 can be improved considerably by proceeding in an analogous but more conservative manner. We record the outcome of the procedure, omitting the details 
COROLLARY 2'. The free metabelian group of rank two can be embedded in a 5-generator metabelian group $H$ which can be defined by 12 defining relations. The generators of $H$ may be chosen to be

$$
u_{1}, u_{2}, t_{1}, t_{2}, a
$$

while the 12 defining relations in terms of these generators may be taken to be

$$
\begin{gathered}
{\left[u_{1}, u_{2}\right]=\left[u_{1}, t_{1}\right]=\left[u_{2}, t_{2}\right]=\left[u_{1}, t_{2}\right]=\left[u_{2}, t_{1}\right]=\left[t_{1}, t_{2}\right]} \\
=\left[a, a^{t_{1}}\right]=\left[a, a^{t_{2}}\right]=1 \\
{\left[a, a^{t_{1} t_{2}}\right]=\left[a^{t_{1}}, a^{t_{2}}\right]=1} \\
a^{u_{1}}=a^{1+t_{1}}, a^{u_{2}}=a^{1+t_{2}} .
\end{gathered}
$$

\section{The third step in the proof}

The construction of $\S 7$ makes it easy to complete the proof of the main result of this paper. In order to do so we need to invoke the following

Lemma 7. Let $M$ be a finitely generated $\mathbb{Z} T$-module. If $t$ is an element of infinite order in $T$ then there exists an

$$
\begin{gathered}
f=1+c_{1} t+\cdots+c_{s-1} t^{s-1}+t^{s}(s \geqq 1) \text { in } \mathbb{Z} T \text { such that } \\
m f=0 \text { only if } m=0(m \in M) .
\end{gathered}
$$

Proof. We term a monic polynomial in $t$ of degree at least 1 with constant term 1 special. Let

$$
M_{1}=\{m \in M \mid m h=0 \text { for some special polynomial } h\} .
$$

Now $\mathbb{Z} T$ is a commutative ring and the product of special polynomials is special. It follows readily from these remarks that $M_{1}$ is a submodule of $M$. Since $T$ is a finitely generated abelian group, $\mathbb{Z} T$ is a Noetherian ring. So $M_{1}$ is a finitely generated module because $M$ is (see e.g. Atiyah and Macdonald [5], Chapter 7). Suppose $M_{1}$ is generated by

$$
z_{1}, z_{2}, \cdots, z_{q}(q<\infty) .
$$

Since $z_{i} \in M_{1}$ there is a special polynomial, say $p_{i}$, such that $z_{i} p_{i}=0$. Put

$$
p=p_{1} p_{2} \cdots p_{q} .
$$

Remembering again that $\mathbb{Z} T$ is commutative it follows that

$$
z_{i} p=0(i=1,2, \cdots, q) .
$$

The polynomial $p$ is special, since it is a product of special polynomials. It follows immediately that 
is also special.

$$
f=1+p t
$$

Suppose now that $m \in M$ and $m f=0$. Then, by the very definition of $M_{1}$, $m \in M_{1}$. So $m p=0$. Thus

$$
0=m f=m(1+p t)=m+m p t=m \text {. }
$$

This completes the proof of Lemma 7.

We proceed now to the third step in the proof of the main theorem. Thus suppose $G$ is a finitely generated metabelian group. By Lemma 3 there exists a free abelian group $A$ of finite rank and a finitely generated abelian group $T$ such that $G$ is isomorphic to a subgroup of $W / N$, where $W=A \nmid T$ and $N$ is a normal subgroup of $W$ contained in the base group $B$ of $W$. As in $\S 8$ we put $M=B / N$, and view it as an $R(=\mathbb{Z} T)$-module. We now choose, by Lemma 7, a special polynomial $f_{i}$ in $t_{i}(i=1,2, \cdots, k)$ such that

$$
m f_{i}=0 \text { only if } m=0(m \in M) .
$$

We then put

$$
F=\left\{f_{1}, f_{2}, \cdots, f_{k}\right\}
$$

This means that $F$ satisfies the condition (20) in $\$ 7$. Therefore we may proceed, as in $\S 7$, to the construction of the group denoted $E$ and the homomorphism $\psi$ (see $\S 7$ ). Now this homomorphism $\psi$ maps $W_{F}=W^{*}$ onto $E$. Hence $E$ is a quotient of a finitely presented metabelian group and so is also finitely presented, as we remarked already in $\S 2$. Since $G$ is embedded in $W / N$ and $W / N$ is itself embedded in $E, G$ is embedded in the finitely presented metabelian group $E$. This completes the proof of the theorem.

\section{Some concluding remarks}

Just how close is the connection between a finitely generated metabelian group and the finitely presented metabelian groups that contain it, remains unclear. However the methods of this paper seem likely to provide us with a great deal more information about the complexity of finitely presented solvable groups. In fact it follows that there exist finitely presented solvable groups which are not residually finite. This answers a question that has apparently been open for some time. The details will appear elsewhere.

\section{References}

[1] G. Higman, 'Subgroups of finitely presented groups', Proc. Roy. Soc. London Ser. A 262 (1961), 455-475.

[2] P. Hall, 'Finiteness conditions for soluble groups', Proc. London Math. Soc. (3) 4(1954), 419-436. MR 17, 344. 
[3] G. Baumslag, 'A finitely presented metabelian group with a free abelian derived group of infinite rank', Proc. Amer. Math. Soc. 35 (1972), 61-62.

[4] W. Magnus, 'On a theorem of Marshall Hall', Ann. Math. 40 (1939), 764-768.

[5] M. F. Atiyah and I. G. Macdonald, Introduction to commutative algebra (Addison - Wesley, 1969) ME 30 \# 4129.

Rice University

Houston, Texas

U.S.A.

Present address:

City College of the City University of New York

U.S.A. 